\title{
International Conference on Natural Fibers-Sustainable Materials for Advanced Applications 2013
}

\author{
Raul Fangueiro, ${ }^{1}$ R. Alagirusamy, ${ }^{2}$ Amar Mohanty, ${ }^{3}$ \\ Hu Hong, ${ }^{4}$ and António Torres Marques ${ }^{5}$ \\ ${ }^{1}$ Department of Civil Engineering, University of Minho, Guimarães, Portugal \\ ${ }^{2}$ Department of Textile Technology, Indian Institute of Technology, Delhi, India \\ ${ }^{3}$ Department of Plant Agriculture, School of Engineering, University of Guelph, Guelph, Canada \\ ${ }^{4}$ Institute of Textiles and Clothing, The Hong Kong Polytechnic University, Hong Kong \\ ${ }^{5}$ Department of Mechanical Engineering, University of Porto, Porto, Portugal
}

Correspondence should be addressed to Raul Fangueiro; rfangueiro@civil.uminho.pt

Received 8 December 2013; Accepted 8 December 2013

Copyright (C) 2013 Raul Fangueiro et al. This is an open access article distributed under the Creative Commons Attribution License, which permits unrestricted use, distribution, and reproduction in any medium, provided the original work is properly cited.

Natural fibers are a renewable resource par excellencethey have been renewed by nature and human ingenuity for millennia. Besides, they are carbon neutral once they absorb the same amount of carbon dioxide which they produce. During processing, natural fibers generate mainly organic wastes and leave residues that can be used to generate energy or make ecological housing material. And, at the end of their life cycle, they are $100 \%$ biodegradable.

Natural fibers have widely enlarged their range of applications due to the intensive scientific and technical research undertaken by several institutes all over the world, turning these amazing materials into an alternative feasible choice for many situations in civil engineering, medicine, sports, architecture, and design, among others.

The 1st international conference on natural fiberssustainable materials for future applications (ICNF2013) discussed all these new advances and the potential developments by bringing together companies, universities, research and technological centers, and entrepreneurs.

The proceedings of ICNF2013 are the most important scientific result of the event presenting several up-to-date papers on the following topics:

(i) animal and plant based natural fibers;

(ii) fibers produced based on natural resources-Innovative and functional natural fibers;

(iii) nanodimensional natural fibers (e.g., nanocellulose); (iv) properties and characterization of natural fibers;

(v) new, efficient, and cost effective processes for natural fiber extraction and processing;

(vi) natural fiber modification techniques;

(vii) textile processing of natural fibers (spinning, weaving, coloration, finishing, etc.);

(viii) advanced fibrous structures based on natural fibers;

(ix) natural fiber based polymeric and cementitious composites;

(x) green composites;

(xi) applications of natural fibers in high-end sectors (civil engineering, medicine, transportation systems, sports, etc.);

(xii) innovative applications of natural fibers;

(xiii) product design with natural fibers;

(xiv) modelling and prediction of properties and behaviour.

Raul Fangueiro R. Alagirusamy Amar Mohanty Hu Hong António Torres Marques 


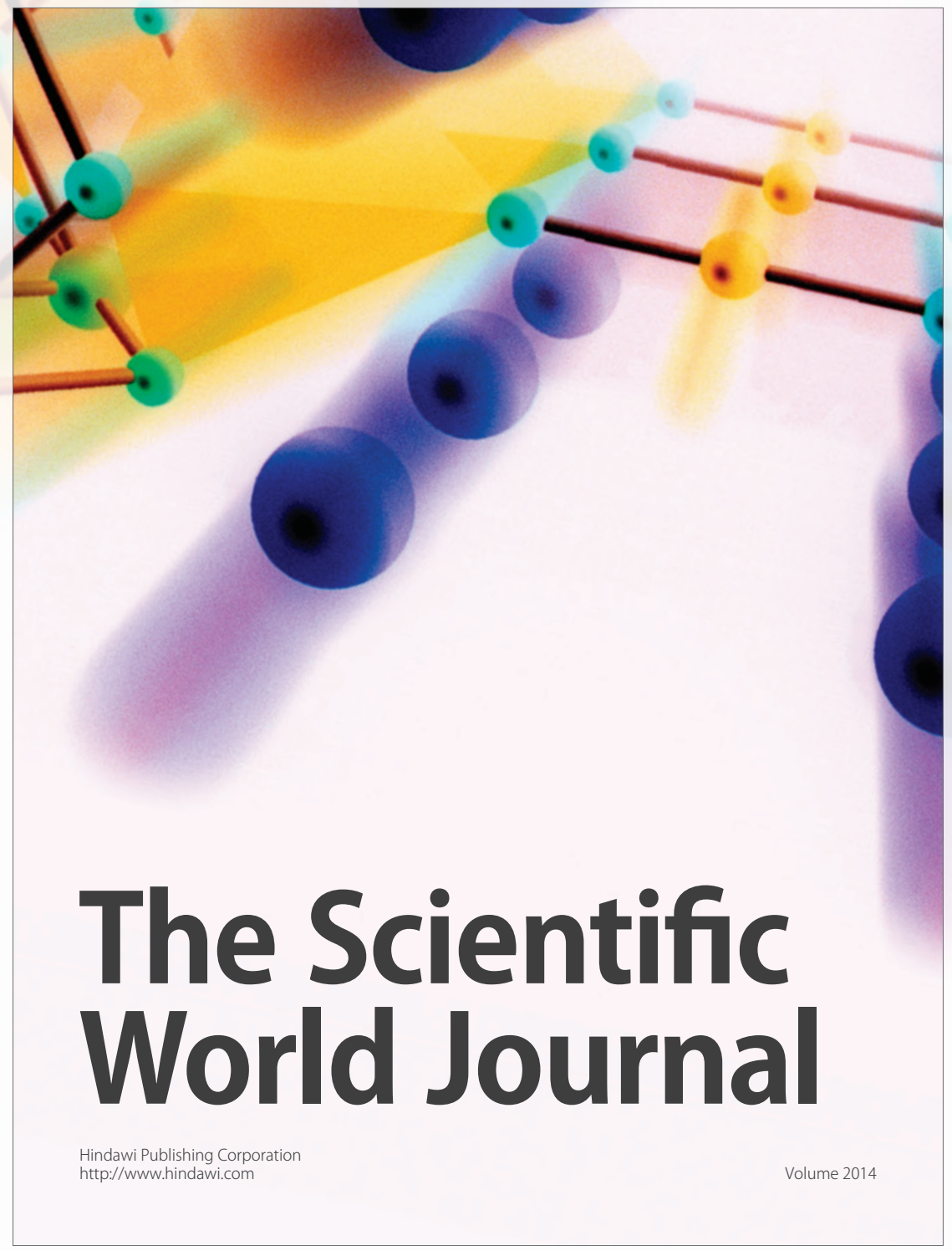

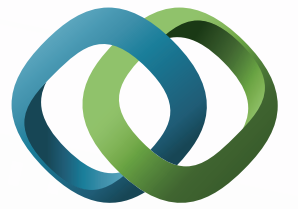

Hindawi
- Impact Factor $\mathbf{1 . 7 3 0}$

- 28 Days Fast Track Peer Review

- All Subject Areas of Science

- Submit at http://www.tswj.com 

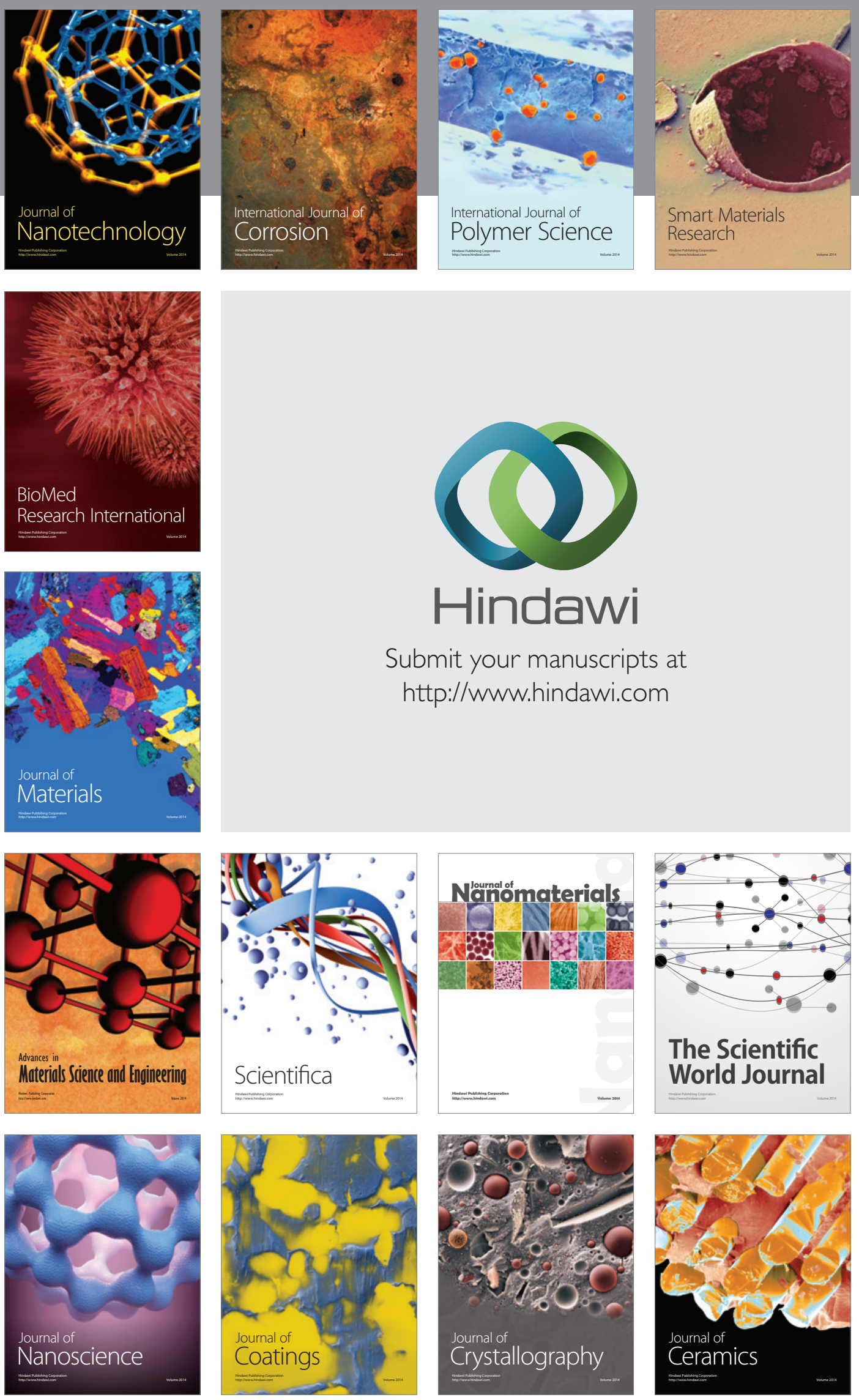

The Scientific World Journal

Submit your manuscripts at

http://www.hindawi.com

\section{World Journal}

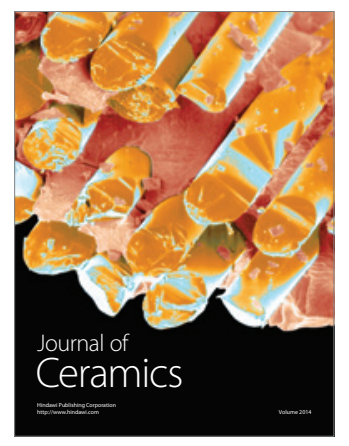

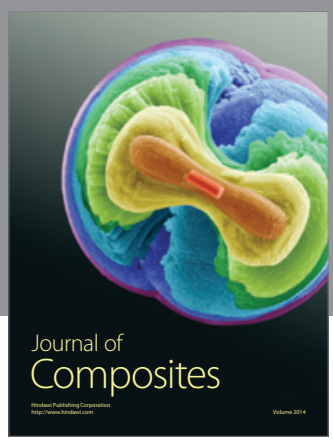
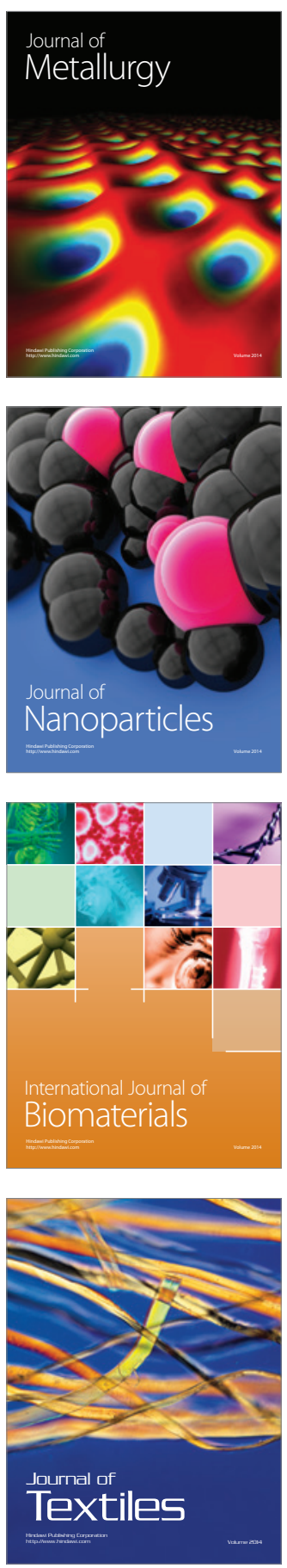